\title{
Identification of single flexible-joint robot dynamics: a nonparametric approach
}

\author{
Bassem Boukhebouz \\ CEA LIST and University of Strasbourg \\ Interactive Robotics Laboratory \\ and ICube Laboratory \\ Palaiseau, France \\ bassem.boukhebouz@cea.fr \\ Xavier Lamy \\ CEA LIST \\ Interactive Robotics Laboratory \\ Palaiseau, France \\ xavier.lamy@cea.fr
}

\author{
Guillaume Mercère \\ University of Poitiers \\ LIAS Laboratory \\ Poitiers, France \\ guillaume.mercere@univ-poitiers.fr
}

\author{
Mathieu Grossard \\ CEA LIST \\ Interactive Robotics Laboratory \\ Palaiseau, France \\ mathieu.grossard@cea.fr
}

\author{
Édouard Laroche \\ University of Strasbourg \\ ICube Laboratory \\ Strasbourg, France \\ laroche@unistra.fr
}

\begin{abstract}
In this paper, a nonparametric identification method is used to identify the dynamic model of a flexible joint robot (FJR) that includes friction and transmission nonlinearities. More precisely, the Best Linear Approximation (BLA) approach has been considered in a closed-loop setup to provide the frequency response of the system and an evaluation of the nonlinearities. Based on simulated data, the paper investigates the tuning of an adequate multisine excitation signal that enables good identification results. First, an algorithm is adapted in order to shape the spectrum of the control signal adequately. Second, based on an evaluation of the effects on the Coulomb friction and transmission nonlinearity, it is showed that an optimal amplitude can be found.
\end{abstract}

Index Terms-System identification, frequency domain, nonlinear distortions, flexible joint robots.

\section{INTRODUCTION}

When manipulator robots are used on large workspace with fast dynamical changes, nonlinear behavior may occur [1]. This is all the more true when these robotic systems have strong flexibilities (like structural [2] or joint flexibilities [3]) because of $(i)$ the coupling between the rigid bodies and elastic deformations of joints, (ii) position dependent nonlinearities such as the well known Coulomb friction [4] or (iii) variable stiffness due to motor-to-joint transmission [5] to name a few. White box models of such nonlinear systems can be generated based on mechanical modeling of rigid and/or flexible multibodies chains. Because such an approach requires an accurate knowledge of the robot under study as well as high level skills in robotics, system identification [6] is often suggested as a valuable data based tool to bypass these modeling difficulties. In order to benefit from the white box analysis in the system identification procedure, parametric models are often used for modeling manipulator robots [1], [7], [8], [9]. This kind of gray box model often neglects strong nonlinearities (for example the stiffness of the transmission) in order to keep, in the end, a model structure $(i)$ generic to mimic the system behavior, (ii) simple enough to ensure that the controller can be designed easily. Neglecting the effect of nonlinearities on resonance phenomena may unfortunately lead to conservative low-bandwidth controllers or simplified models which do not involve the resonance phenomena (this can damage the system if such phenomena occur within the control bandwidth). This is one of the main reasons why, at a first step, it is often suggested performing a nonparametric identification of the flexible joint robot [10], mainly in the frequency domain, in order to provide knowledge of the input-output behavior of the system without requiring strong structural knowledge of the robotic system to control. By resorting to a divide and conquer strategy, nonparametric frequency response function (FRF) identification algorithms can be used locally to determine linear models of the robot behavior in various configurations around user defined working points.

This paper is dedicated to the nonparametric estimation of the FRF of FJRs in the presence of friction and transmission nonlinearities using the Best Linear Approximation -BLAmethod described in details in [11] and [12]. Our contributions firstly consist in adapting the multisine amplitudes by restoring to an iterative procedure which shapes the multisine spectrum in order to optimize the quality of the identified model. Furthermore, by applying odd random phase multisine as excitation signal in a closed loop setup, we determine the BLA of the underlying linear transfer from the motor torque to the motor position, the variance of the stochastic nonlinear contributions as well as the noise variance. Secondly, the impact of the nonlinear nature of the transmissions is revealed by mapping the resonances and antiresonances frequencies of the FRF to the levels of excitation signals.

The paper is organized as follows. In Section II, the modeling of the flexible joint robot is introduced. Section 
III is dedicated to the basics of the frequency response function estimation with the BLA approach. In Section IV, we investigate the tuning of the multisine excitation signal and we show the simulation results of the identification of FJR. Concluding remarks are provided in section V.

\section{Modeling of Flexible Joint Robot Dynamics}

When it comes to the compliant manipulation in contact with human, flexible joint robots are more suitable and provide better performance than rigid robots. By FJRs, we refer to robots in which the flexibility is located at the articulation level [3]. In this section, we first present the mathematical description of a FJR with $n$ degrees of freedom. Then, the modeling of single FJR is discussed along with the description of nonlinearities considered in the current work.

The most commonly used model to describe the dynamics of FJRs is called the reduced model proposed by [3]. In this model, the inertial coupling between the motors and the links of the robot is neglected. The dynamics of the robot typically consist of two coupled equations as follows

$$
\begin{aligned}
M(q) \ddot{q}+C(q, \dot{q}) \dot{q}+\tau_{G}(q)+\tau_{f_{l}}(\dot{q})+\tau_{e}(q, \theta) & =0, \\
J_{m} \ddot{\theta}+\tau_{f_{m}}(\dot{\theta})-\tau_{e}(q, \theta) & =\tau,
\end{aligned}
$$

with $q$ is the joint position, while $\theta$ is the motor position. $M(q)$ represents the inertia matrix of the rigid bodies of the poly-articulated chain. $J_{m}$ denotes the inertia matrix of the actuators, $C(q, \dot{q}) \dot{q}$ is the Coriolis torques, $\tau_{G}(q)$ is the gravity torque, $\tau_{f_{l}}(\dot{q})$ and $\tau_{f_{m}}(\dot{\theta})$ are the friction torques on the link side and motor side, respectively, while $\tau_{e}(q, \theta)$ is the elastic torque.

The friction torques on the link and the motor side according to Coulomb friction law are given by

$$
\begin{aligned}
\tau_{f_{l}} & =f_{v} \dot{q}+f_{s} \operatorname{sgn}(\dot{q}), \\
\tau_{f_{m}} & =f_{v m} \dot{\theta}+f_{s m} \operatorname{sgn}(\dot{\theta}),
\end{aligned}
$$

where $f_{v}$ and $f_{s}$ are the viscous and dry friction coefficients on the joint side, respectively, while $f_{v m}$ and $f_{s m}$ are the viscous and dry friction coefficients on the motor side, respectively.

The flexibility at the joint level transforms the deflection $q-\theta$ into an elastic torque as follows

$$
\tau_{e}=K(q, \theta)(q-\theta)
$$

where the stiffness $K(q, \theta)$ of the transmission is deflection dependent. This stiffness can be a nonlinear polynomial function of order $n$ according to [5]. In our work, we consider the case of a second order polynomial stiffness as given below, where $k_{0}$ and $k_{1}$ are stiffness coefficient, i.e.,

$$
K(q, \theta)=k_{0}+k_{1}(q-\theta)^{2} .
$$

Consider a single FJR on a horizontal plane, hence, without gravity (see Fig. 1). When the friction torques as well as the elastic torque given by Eq. (2) and Eq. (3), respectively, are included in Eq. (1), the dynamical model is

$$
\begin{array}{r}
J \ddot{q}+f_{v} \dot{q}+f_{s} \operatorname{sgn}(\dot{q})+k_{0}(q-\theta)+k_{1}(q-\theta)^{3}=0, \\
J_{m} \ddot{\theta}+f_{v m} \dot{\theta}+f_{s m} \operatorname{sgn}(\dot{\theta})+k_{0}(\theta-q)+k_{1}(\theta-q)^{3}=\tau .
\end{array}
$$

Eq. (5) is nonlinear, coupled and characterized by elastic modes. We consider the case where only the motor position $\theta$ is available, and we locally calculate the underlying linear transfer function from the motor torque to the motor position $(\tau \rightarrow \theta)$. Such transfer is of prime importance for feedback control design and is known as the collocated transfer [3]. By omitting the nonlinear terms in Eq. (5), we derive the transfer functions of interest, i.e., from the input $\tau$ to the output $\theta$, we get

$$
G_{0}(s)=\frac{\theta(s)}{\tau(s)}=\frac{J s^{2}+\left(f_{v}+f_{s}\right) s+k_{0}}{\operatorname{den}(\mathrm{s})},
$$

with den(s) given by

$$
\begin{aligned}
\operatorname{den}(\mathrm{s})=\left\{J J_{m} s^{3}+\right. & {\left[J\left(f_{v m}+f_{s m}\right)+J_{m}\left(f_{v}+f_{s}\right)\right] s^{2} } \\
+[(J+ & \left.\left.J_{m}\right) k_{0}+\left(f_{v}+f_{s}\right)\left(f_{v m}+f_{s m}\right)\right] s \\
& \left.+\left[\left(f_{v}+f_{s}\right)+\left(f_{v m}+f_{s m}\right)\right] k_{0}\right\} s .
\end{aligned}
$$

By neglecting the friction terms, $G_{0}(s)$ becomes

$$
G_{0}(s)=\frac{s^{2}+\omega_{a r}^{2}}{s^{2}\left(s^{2}+\omega_{a}^{2}\right)} .
$$

where the antiresonance $\omega_{a r}$ and the resonance $\omega_{r}$ are given by

$$
\omega_{a r}=\sqrt{\frac{k_{0}}{J}}, \omega_{r}=\sqrt{\frac{\left(J+J_{m}\right) k_{0}}{J J_{m}}} .
$$

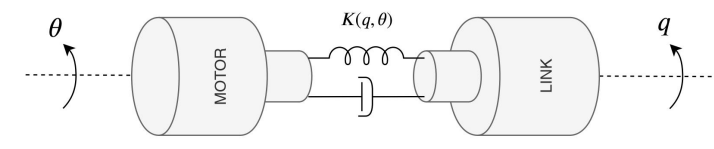

Fig. 1. Single flexible joint.

In practice, calculating $G_{0}(s)$ is not carried out by going through the linearization since the nonlinearities are usually unknown. For this reason, the FRF is a fundamental tool which allows the estimation of $G_{0}(s)$ from the measurement of the input-output signals of the system under study. Such a step is crucial and essential in the identification process because it gives the first insights about the input-output behavior of the system and allows the user to determine the frequency band of interest. In the next section, we present the BLA method [13] to reach this goal. This method enables to accurately estimate the underlying linear system $G_{0}(s)$ in the presence of nonlinearities, to detect and to quantify nonlinear distortions and noise contributions [13]. 


\section{Frequency Response Function Measurement}

In the frequency domain, nonparametric identification consists in estimating the FRF of a system given the measurements of the input $u(t)$ and output $y(t)$ signals by calculating their discrete Fourier transforms -DFTs-. In this section, we introduce a method which allows accurate estimation of a linear model of a nonlinear system. Furthermore, the dedicated tools to minimize the impact of nonlinearities are presented along.

\section{A. Best linear approximation}

For systems that are subject to nonlinear distortions represented by a Volterra series [14] and for a class of Gaussianlike excitations [15], the FRF can be written as [13]

$$
G\left(j \omega_{k}\right)=\hat{G}_{0}\left(j \omega_{k}\right)+G_{s}\left(j \omega_{k}\right)+N_{G}(k),
$$

where $\omega_{k}\left(k=1,2, \ldots, F_{\max }\right)$ stands for the excited frequencies, $\hat{G}_{0}$ is an estimate of the underlying linear model $G_{0}, G_{s}$ is a zero mean stochastic nonlinear distortions and $N_{G}$ represents the noise contributions. It was shown in [13], that a consistent estimation of $G_{0}$, called the best linear approximation $G_{B L A}$, can be obtained using Gaussian-like excitations.

Due to the instability of the model given by Eq. (5), the identification procedure is performed in a closed-loop setup [16] as shown in Fig. 3. The difficulty is that the input $u(t)$ depends on the nonlinear distortions because of the feedback loop, which introduces bias in the estimation of BLA. In [17], this problem was solved by presenting a generalized definition of the BLA. The idea consists in using the BLA via the indirect method [18], where we use the reference signal $r(t)$ (typically the excitation signal), the input signal $u(t)$ and the output signal $y(t)$ to identify two systems, i.e, $G_{r y}\left(j \omega_{k}\right)$ and $G_{r u}\left(j \omega_{k}\right)$, respectively. The BLA is then given by

$$
G_{B L A}\left(j \omega_{k}\right)=\frac{S_{y r}(j \omega)}{S_{u r}(j \omega)},
$$

where $S_{y r}\left(j \omega_{k}\right)$ and $S_{u r}\left(j \omega_{k}\right)$ are the reference-output and the reference-input cross-power spectrum, respectively.

\section{B. Best linear approximation approaches}

Two estimation approaches were presented in [11] and [12] to calculate the BLA of a system in presence of nonlinear distortions:

- The fast approach is based on a single realization of odd random phase multisine excitation given by Eq. (13), i.e., with $M=1$ realization and $P \geq 2$ periods (see Fig. $2(a)$ ). From the excited harmonics of the odd multisine, we randomly eliminate some harmonics called the nonexcited lines (detection lines). The elimination of harmonics consists in $(i)$ gathering the excited odd harmonics into groups of $F_{\text {group }}$ consecutive harmonics (typical values for $F_{\text {group }}$ are two, three, or four [13]) (ii) for each group, one randomly selected harmonic is omitted. For a nonlinear system, even harmonics in the input spectrum show up in the output spectrum at even nonexcited harmonics only, regardless of the parity of the nonlinearity, while the odd harmonics in the input spectrum appear at even and odd nonexcited harmonics in the output spectrum according to the parity of the nonlinearity. The level of the output spectrum at the detection lines corresponds to the stochastic nonlinear distortions at the adjacent excited lines. The detection lines enable the calculation of the variance of nonlinear distortions $\hat{\sigma}_{N L}^{2}$ and the variance of noise contributions $\hat{\sigma}_{\text {noise }}^{2}$ [13].

- In the robust method, the information about the stochastic nonlinear distortions are obtained based on the analysis of the mean and variance of the input/output signals by performing several realizations of random phase multisines. For each new realization, a new odd random phase excitation is generated (see Fig. 2 (b)). It was shown in [13] that the variance of the nonlinear distortions are inversely proportional to $M$ and it is recommended to choose $M \geq 7$ and $P \geq 2$. The comparison between the variance of the nonlinear distortion and the noise variance allows the user to distinguish whether the system behaves nonlinearly or not. In order to reduce the impact of the nonlinear distortions, we increase the number of realizations $M$.

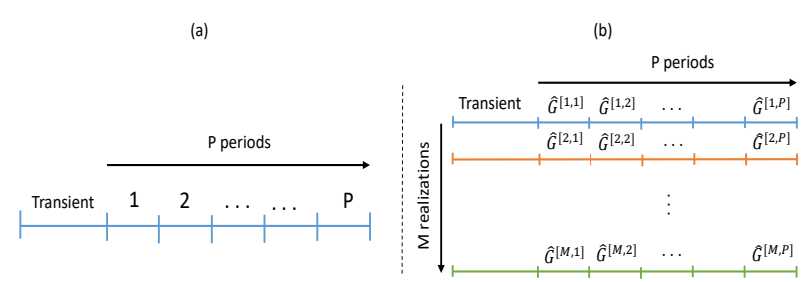

Fig. 2. BLA approaches [13].

In this work, both approaches are used. First, the fast approach is considered for the classification of the nonlinear distortions in even and odd contributions and for the shaping of the excitation spectrum. In a second stage, the robust approach is used to reduce the distortions of the nonlinearities and noise contributions.

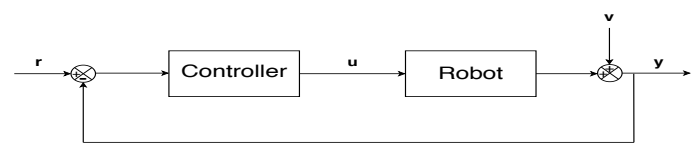

Fig. 3. Closed loop setup.

\section{Best Linear Approximation of Flexible Joint ROBOTS}

In this section, we apply the method described in section III to estimate the FRF of a single FJR from simulated data and to study the impact of the nonlinearities 
on the estimation. The model parameters are: motor inertia $J_{m}=0.2 \mathrm{~kg} \cdot \mathrm{m}^{2}$, link inertia $J=0.3 \mathrm{~kg} \cdot \mathrm{m}^{2}$, parameters of the Coulomb friction $f_{s}=5 \mathrm{~N} . \mathrm{m}, f_{s m}=6 \mathrm{~N} . \mathrm{m}$, $f_{v}=4$ N.m.s.rad ${ }^{-1}, f_{v m}=2$ N.m.s.rad ${ }^{-1}$, deflection dependent stiffness parameters $k_{0}=1500$ N.m.rad ${ }^{-1}$ and $k_{1}=3000$ N.m.rad ${ }^{-2}$. The plant controller $C(s)$ is a parallel PD given by

$$
C(s)=P+D \frac{N}{1+N \frac{1}{s}},
$$

where $P=200$ N.m.rad ${ }^{-1}, D=60$ N.m.s.rad ${ }^{-1}$ and the filter is $N=24$ rad.s $s^{-1}$.

\section{A. Multisine excitation}

From the class of Gaussian-like excitations, the multisine excitation is usually preferred for the estimation of the FRF [19]. The multisine enables $(i)$ to reduce the measurement duration, $(i i)$ to use its periodicity in order to avoid the leakage, (iii) to classify, detect and quantify the nonlinear distortions by a specific choice of the harmonics of the multisine [13]. The multisine excitation is defined as follows

$$
r(t)=\sum_{k=1}^{F_{\max }} A_{k} \cos \left(2 \pi f_{k} t+\phi_{k}\right)
$$

where $A_{k}$ are the amplitudes, $F_{\max }$ is the number of harmonics, $f_{k}$ are integer multiples of the fundamental frequency $f_{0}$ with $k=1,2, \ldots, F_{\max }$ and $\phi_{k}$ are random phases with a uniform distribution in $[0,2 \pi]$. An integer number $P$ of periods of $r(t)$ are acquired after the transient has vanished, thus, providing $N=P N_{\text {period }}$ samples, where $N_{\text {period }}$ is the number of samples over one period.

\section{B. Excitation signal shaping}

In order to evaluate the quality of the estimated FRF and thus to tune the excitation signal accordingly, the following metric has been used that compares the estimated FRF with the actual frequency response of the system $G_{0}$ :

$V=\frac{1}{F_{\max }} \sum_{k=1}^{F_{\max }}\left|20 \log \left(G_{0}\left(j \omega_{k}\right)\right)-20 \log \left(G_{B L A}\left(j \omega_{k}\right)\right)\right|^{2}$.

In experimental conditions, $G_{0}$ is not available. The metric Eq. (14) might be replaced by the variance of the nonlinear distortions $\hat{\sigma}_{N L}^{2}$, to evaluate the quality of the identification. Fig. 4 represents the variations of the metric with respect to the excitation amplitude in four cases: $(i)$ red line: when both nonlinearities given by Eq. (2) and Eq. (3) are included, (ii) blue line: when the transmission nonlinearity is active, (iii) black line: when the friction nonlinearity is used and (iiii) green line: when the data is noisy and both nonlinearities are included. It is clear from Fig. 4 that an optimal amplitude $A_{\text {opt }}$ exists.

Using a flat spectrum on the reference signal results in a non flat spectrum on the control signal, leading to possible saturation and low excitation at some frequencies (see both spectrum on sub-figures (a) and (b) in Fig. 5). In order to get

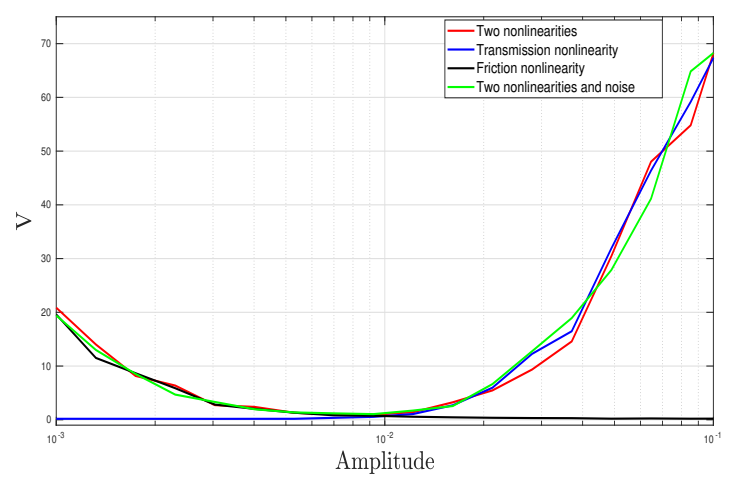

Fig. 4. Variation of the quality of the identification as a function of the excitation amplitude.

a flat spectrum on the control signal, we have used Algorithm 1 that has been adapted from [20]. The results of Algorithm

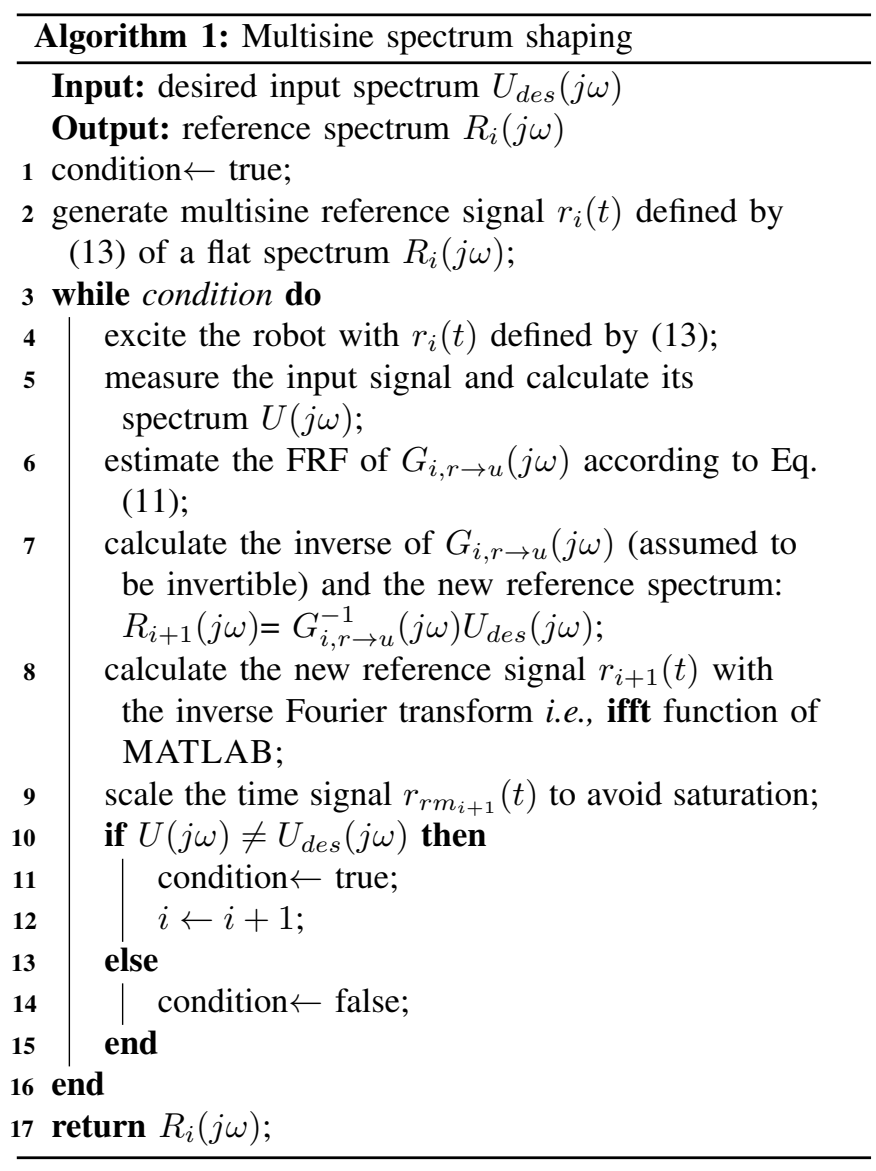

1 are shown in Fig. 5, for an odd random phase multisine, within the band of excitation $0.1 \mathrm{~Hz}-50 \mathrm{~Hz}$ and a frequency resolution $f_{\text {res }}=0.1 \mathrm{~Hz}$. The top of Fig. 5, i.e., $(a)$ and (b), represents the case without the input shaping and the bottom, i.e., $(c)$ and $(d)$ with the use of the input shaping. Excited odd harmonics (in black), non-excited odd harmonics (in red) and non-excited even harmonics (in blue) are shown. 


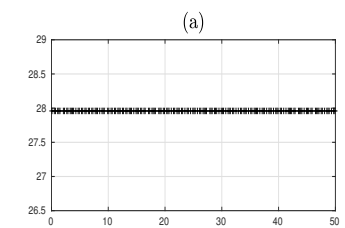

(c)
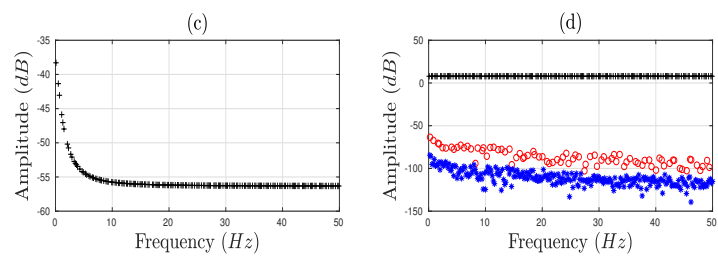

Fig. 5. Reference (multisine) and input (motor torque) spectrum.

Whereas, only some odd harmonics are excited (in black in subfigures (a) and (c)), the energy is also observed on the control signal at the nonexcited odd harmonics, which confirms according to [13] that the system is nonlinear and contains almost one odd nonlinearity. Moreover, the spectrum of the input becomes flat as desired (see Fig. $5(d)$ ). By using Algorithm 1, the metric Eq. (14) on the quality of the estimation at the optimal amplitude was reduced from $0.94 d B$ to $0.78 d B$.

\section{Nonlinearity impact on the FRF estimate}

The impacts of the friction and nonlinear transmission on the FRF estimate are first evaluated separately and then together. The data is affected by a Gaussian zero mean white noise of $S N R=20 d B$.
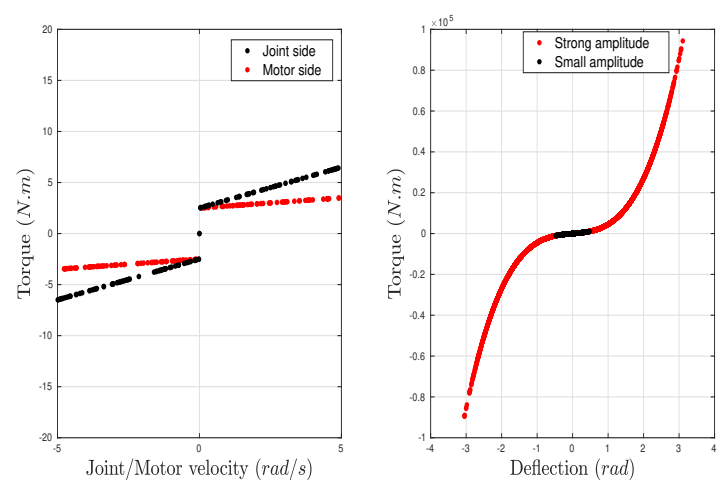

Fig. 6. Friction and elastic torques characteristics.

\section{Friction nonlinearity impact:}

As can be seen on the black line in Fig. 4, the friction nonlinearity mostly introduces errors in the estimation for small amplitudes. Thus, to study the friction impacts, we choose a small excitation amplitude $\left(A_{k}=10^{-3} \mathrm{rad}\right)$ and we consider that the stiffness is linear (i.e., $k_{1}=0$ ). As a result, the only acting nonlinearity is the Coulomb friction (see Fig. 6 (left)). In the first instance, the FRF is estimated by making a single realization $(M=1$ and $P=7)$. The resulting FRF estimate is shown in Fig. 7. We observe that the Coulomb friction nonlinear distortions are indeed present and cause larger variability in the FRF (resonances and antiresonances are masked). This is sensible because the dry friction acts as a damping.

In order to reduce these nonlinear distortions and improve the quality of the estimation, 40 realizations ( $M=40$ and $P=4$ ) are performed, because increasing the number of periods $P$ reduces the noise contribution only on the FRF estimate [13]. By averaging 40 realizations, these distortions are reduced. The plot in black in Fig. 9. reveals that the best linear approximation corresponds (almost) to the linearized model.

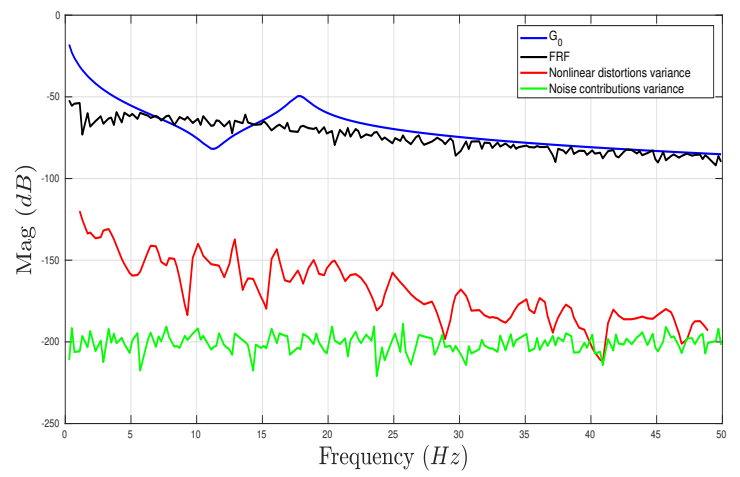

Fig. 7. Fast approach $(M=1$ and $P=7$ ) for estimating the gain of FRF between $\tau$ and $\theta$ in the presence of friction nonlinearity. The gain of $G_{B L A}$ (in black) is compared with the gain of the linearized model $G_{0}$ (in blue), variance of nonlinear distortions (in red) and variance of noise contributions (in green).

\section{Stiffness nonlinearity impact}

In this case, we increase the value of the excitation amplitude $\left(A_{k}=9 \cdot 10^{-2} \mathrm{rad}\right)$, such that the nonlinearity of the transmission is active (see Fig. 6). In addition, we omit the nonlinear terms in the friction torques, by setting the Coulomb friction coefficients to zero $\left(f_{s}=f_{s m}=0\right)$. As a result, the only source of nonlinearity is the deflection depending stiffness. First, the FRF is estimated by making a single realization $(M=1$ and $P=7)$. The results are shown in Fig. 8. We observe that the nonlinear distortions are larger than the measurement noise contributions (variance of noise contributions $\hat{\sigma}_{\text {noise }}^{2}$ is at least $100 d B$ lower than the variance of the nonlinear distortions $\hat{\sigma}_{N L}^{2}$. Thus, these fluctuations in the FRF are mostly due to the nonlinear distortions of the transmission.

By performing 100 realizations, we reduce the transmission nonlinear distortions via the robust method (see the result in green in Fig. 9). The resonance and anti-resonance frequencies are stiffness dependent according to Eq. (9), thus, for high amplitudes they are shifted to the right.

\section{Friction and stiffness nonlinearities impact:}

The optimal amplitude $\left(A_{\text {opt }}=8 \cdot 10^{-3} \mathrm{rad}\right)$ from Fig. 


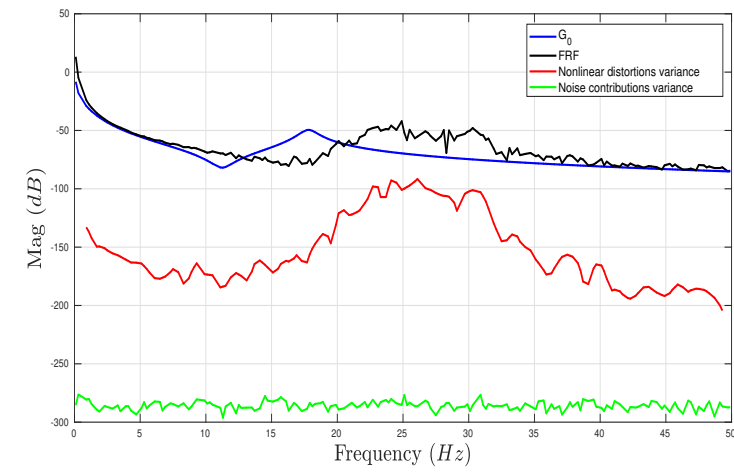

Fig. 8. Fast approach $(M=1$ and $P=7$ ) for estimating the gain of FRF between $\tau$ and $\theta$ in the presence of transmission nonlinearity. The gain of $G_{B L A}$ (in black) is compared with the gain of the linearized model $G_{0}$ (in blue), variance of nonlinear distortions (in red) and variance of noise contributions (in green).

4 is used to estimate the FRF in the presence of both nonlinearities. Fig. 9 in red, shows that, for the optimal amplitude and even in the presence of both nonlinearities, the FRF estimation via the robust method fits $G_{0}$ perfectly.

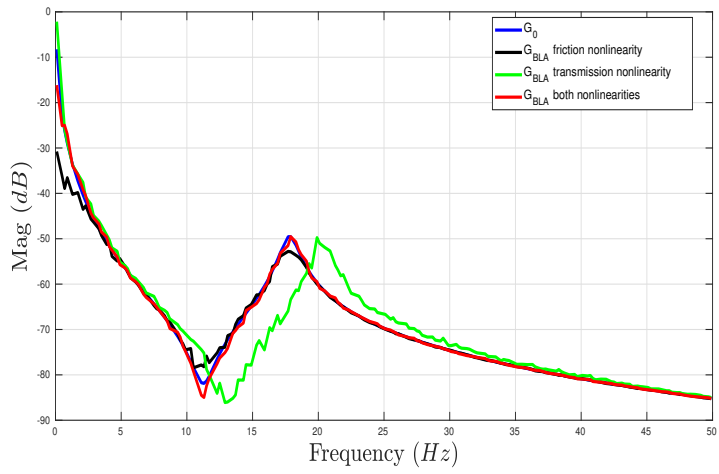

Fig. 9. Robust approach for estimating the gain of FRF between $\tau$ and $\theta$.

\section{CONClusion And Perspectives}

In this paper, a nonparametric approach based on the BLA method has been investigated to estimate the FRF of a single FJR, in the presence of Coulomb friction and transmission nonlinearities, in a closed-loop setup. To get an accurate FRF estimate and to reduce the nonlinear distortions, several experiments were performed with different phase initializations of the multisine excitation signal. Our investigations have highlighted the dependence of the estimation on the signal amplitude. Moreover, an optimal amplitude could be found, that makes a trade-off in terms of quality of the identified model between the effects of the Coulomb friction and the nonlinearity of the transmission. Future work will consist in using the BLA method on an experimental data, in order to detect nonlinearities and accurately estimate the FRF of a flexible joint robot.

\section{REFERENCES}

[1] W. Khalil and E. Dombre, Modeling, identification and control of robots. Butterworth-Heinemann, 2004.

[2] G. Mercère, M. Lovera, and E. Laroche, "Identification of a flexible robot manipulator using a linear parameter-varying descriptor statespace structure," in Proceedings of the 50th IEEE Conference on Decision and Control and European Control Conference, Dec 2011, pp. 818-823.

[3] A. De Luca and W. Book, Robot with flexible elements, in Springer Handbook of Robotics, edited by B.Siciliano and O. Khatib, Springer, p.287-319, B.Siciliano and O. Khatib, Eds. Springer Handbook of Robotics, 2008.

[4] P. Hamon, M. Gautier, and P. Garrec, "New dry friction model with load- and velocity-dependence and dynamic identification of multidof robots," in Proceedings of the IEEE International Conference on Robotics and Automation, May 2011, pp. 1077-1084.

[5] F. Fichera and M. Grossard, "On the modeling and identification of stiffness in cable-based mechanical transmissions for robot manipulators," Mechanism and Machine Theory, vol. 108, pp. 176 - 190, 2017.

[6] L. Ljung, System Identification: Theory for the User. Upper Saddle River, NJ, USA: Prentice-Hall, Inc., 1986.

[7] M. Gautier, "Dynamic identification of robots with power model," in Proceedings of the International Conference on Robotics and Automation, vol. 3, april 1997, pp. 1922-1927.

[8] E. Wernholt and S. Moberg, "Frequency-domain gray-box identification of industrial robots," IFAC Proceedings Volumes, vol. 41, no. 2 , pp. 15372 - $15380,2008$.

[9] M. Östring, S. Gunnarsson, and M. Norrlöf, "Closed-loop identification of an industrial robot containing flexibilities," Control Engineering Practice, vol. 11, no. 3, pp. 291 - 300, 2003.

[10] E. Wernholt and S. Gunnarsson, "Estimation of nonlinear effects in frequency domain identification of industrial robots," IEEE Transactions on Instrumentation and Measurement, vol. 57, no. 4, pp. 856-863, 2008.

[11] R. Pintelon, G. Vandersteen, Ludwig De Locht, Y. Rolain, and J. Schoukens, "Experimental characterization of operational amplifiers: a system identification approach-part i: theory and simulations," IEEE Transactions on Instrumentation and Measurement, vol. 53, no. 3, pp. 854-862, June 2004.

[12] R. Pintelon, Y. Rolain, G. Vandersteen, and J. Schoukens, "Experimental characterization of operational amplifiers: a system identification approach-part ii: calibration and measurements," IEEE Transactions on Instrumentation and Measurement, vol. 53, no. 3, pp. 863-876, June 2004.

[13] R. Pintelon and J. Schoukens, System identification: a frequency domain approach. John Wiley \& Sons, 2012.

[14] M. Schetzen, The Volterra and Wiener Theories of Nonlinear Systems. Melbourne, FL, USA: Krieger Publishing Co., Inc., 2006.

[15] J. Schoukens, J. Lataire, R. Pintelon, and G. Vandersteen, "Robustness issues of the equivalent linear representation of a nonlinear system," in Proceedings of the IEEE Instrumentation and Measurement Technology Conference, May 2008, pp. 332-335.

[16] P. Van den Hof, "Closed-loop issues in system identification," Annual reviews in control, vol. 22, pp. 173-186, 1998.

[17] R. Pintelon and J. Schoukens, "Frf measurement of nonlinear systems operating in closed loop," IEEE Transactions on Instrumentation and Measurement, vol. 62, no. 5, pp. 1334-1345, May 2013

[18] T. Söderström and P. Stoica, Eds., System Identification. Upper Saddle River, NJ, USA: Prentice-Hall, Inc., 1988.

[19] J. Schoukens, R. Pintelon, E. van der Ouderaa, and J. Renneboog, "Survey of excitation signals for fft based signal analyzers," IEEE Transactions on Instrumentation and Measurement, vol. 37, no. 3, pp. 342-352, Sep. 1988.

[20] F. Saupe and A. Knoblach, "Design of excitation signals for the closed loop identification of industrial robots," in Proceedings of the IEEE International Conference on Control Applications, Oct 2012, pp. 1553 1560. 